\title{
Internal carotid artery surgical revascularization in a pediatric patient with Schimke immuno-osseous dysplasia
}

\author{
Erick M. Westbroek, MD, Nitin Mukerji, MD, Paul Kalanithi, MD, and Gary K. Steinberg, MD, PhD \\ Department of Neurosurgery and Stanford Stroke Center, Stanford University School of Medicine, Stanford, California
}

Schimke immuno-osseous dysplasia (SIOD) is a rare autosomal recessive disorder characterized by spondyloepiphyseal dysplasia, episodic lymphopenia, renal failure, and cerebrovascular disease secondary to arteriosclerosis and myointimal hyperplasia. In this paper the authors report the first known application of internal carotid artery (ICA) surgical revascularization to relieve a high-grade focal stenosis of the ICA in a pediatric patient, a 6 -year-old boy with SIOD. The clinical presentation, imaging features, operative technique, and postoperative course are described and the molecular genetics, pathophysiology, and treatment considerations in SIOD are discussed.

http://thejns.org/doi/abs/10.3171/2014.10.PEDS14141

KEY WORDS carotid endarterectomy; dysplasia; vascular disorders

$\mathrm{S}$ CHIMKE immuno-osseous dysplasia (SIOD) is a rare autosomal recessive disorder characterized by growth retardation from spondyloepiphyseal dysplasia, T-cell immunodeficiency secondary to episodic lymphopenia, pigmentary skin changes, and progressive renal dysfunction. Arteriosclerotic changes are another key feature of the clinical syndrome and are associated with the moyamoya phenomenon, transient cerebral ischemia, and cerebral infarction commonly observed in these patients..$^{2,5,7}$ Life expectancy of severely affected individuals with SIOD typically does not exceed 1 decade, as these patients usually succumb to renal failure or infection. ${ }^{3}$ In this paper we report a pediatric case of internal carotid artery (ICA) stenosis secondary to SIOD treated by carotid arteriotomy and patching. To our knowledge this represents the first reported case of carotid patching and revascularization in a pediatric patient.

\section{Case Report}

Presentation and Examination

A 6-year-old right-handed boy with SIOD on peritoneal dialysis secondary to end-stage renal disease and bilateral nephrectomy underwent MR angiography and MRI to assess the extent of cerebrovascular disease. The findings suggested left-sided carotid stenosis and multiple small, prior, white matter infarcts in the border zones of the left anterior, middle, and posterior cerebral arteries (Fig. 1).

A CT angiogram confirmed a high-grade stenosis of the left cervical ICA approximately $1 \mathrm{~cm}$ distal to the bifurcation (Fig. 2). The patient was not experiencing neurological symptoms, nor had he experienced them in the past. His neurological examination was normal. We decided to perform a left carotid surgical revascularization to minimize his risk of future stroke.

\section{Operation}

The procedure was performed using electrophysiological monitoring. The patient was positioned, prepared, and draped in the usual fashion for a carotid endarterectomy. Upon identification of the carotid sheath, the common carotid artery was first carefully dissected and the vessel was secured with umbilical tape. Great care was taken not to damage critical surrounding structures. The ICA was dissected superiorly for a distance of approximately $3 \mathrm{~cm}$. After Doppler ultrasonography demonstrated patency of the distal ICA, heparin was given and propofol was administered to achieve burst suppression. Mean arterial blood pressure was increased from 70 to $80 \mathrm{~mm} \mathrm{Hg}$. The

ABBREVIATIONS ICA = internal carotid artery; SIOD = Schimke immuno-osseous dysplasia

SUBMITTED April 18, 2014. ACCEPTED October 16, 2014.

INCLUDE WHEN CITING Published online November 28, 2014; DOI: 10.3171/2014.10.PEDS14141.

DISCLOSURE Dr. Steinberg is a member of the Medtronic Neuroscience Strategic Advisory Board. This work was supported in part by funding from Bernard and Ronni Lacroute, the William Randolph Hearst Foundation, and Russell and Beth Siegelman (to Dr. Steinberg). 

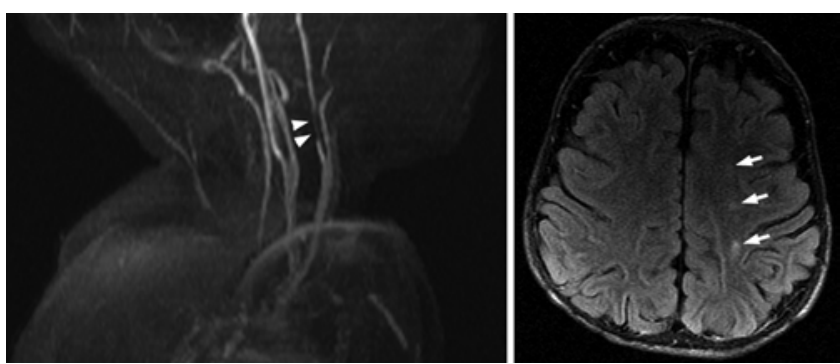

FIG. 1. Left: Screening MR angiogram shows narrowing of the left proximal ICA approximately $1 \mathrm{~cm}$ in length (arrowheads). Right: Axial T2-weighted fluid attenuated inversion recovery (FLAIR) imaging shows multiple hyperintense spot lesions in the white matter of the superior left frontal and parietal lobes, consistent with prior ischemic injury (arrows). The patient was asymptomatic at the time of presentation and had never experienced neurological symptoms.

external carotid artery, distal ICA, and common carotid artery were occluded with aneurysm clips. An arteriotomy was made adjacent to the bifurcation in the proximal ICA. The artery appeared normal here and had a normal luminal diameter. As the arteriotomy was extended distally, thickened tissue was encountered. Myointimal hyperplasia of the proximal and mid left-cervical ICA was found. This caused an approximately $85 \%$ stenosis of the proximal ICA. The arteriotomy was continued past the distal end of the stenotic area. At this point, the clip on the ICA was temporarily released to confirm back bleeding. The external carotid artery was flushed and re-occluded. A Hemashield cardiovascular knitted patch graft (Maquet Getinge Group) was fitted to the arteriotomy opening and sewn in place with 8-0 Prolene suture. The occluding aneurysm clips were removed. Intraoperative Doppler ultrasonography demonstrated patency of all vessels. Meticulous hemostasis was obtained. After 10 minutes had

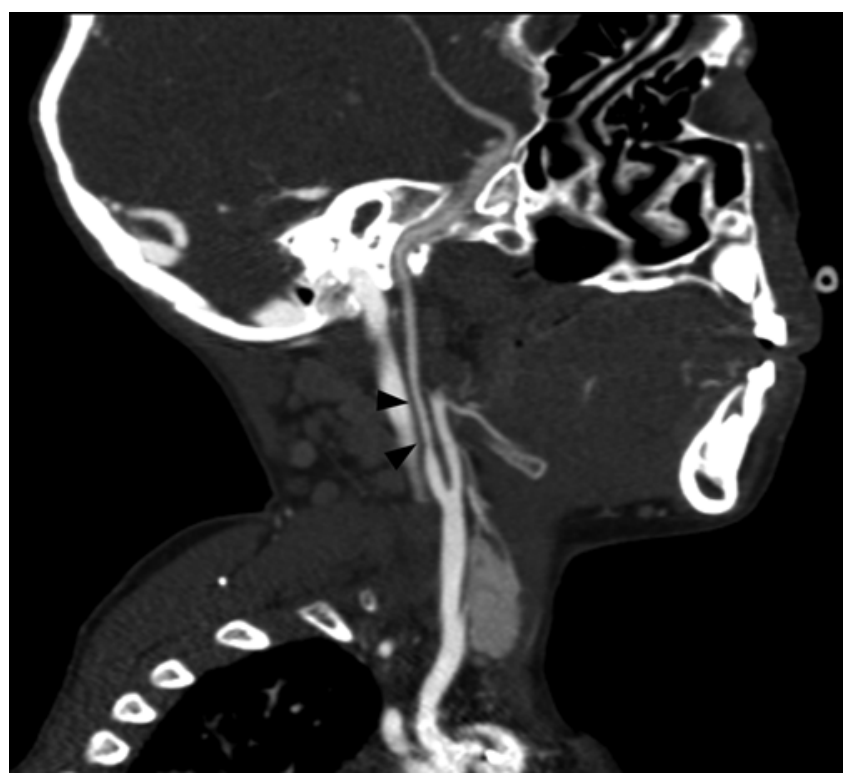

FIG. 2. Sagittal CT angiogram obtained to further delineate MRI findings shown in Fig. 1. The left ICA demonstrates a focal stenosis, just above the carotid bifurcation at approximately the $\mathrm{C}-3$ vertebral body level (arrowheads). Superior to the focal narrowing, the cervical ICA appears diffusely narrow. passed, heparin was partially reversed and the surgical site was closed in the usual fashion. No abnormal changes in electrophysiology were noted over the course of the operation. The patient was extubated and taken to the pediatric intensive care unit in satisfactory condition.

\section{Postoperative Course}

The patient tolerated the procedure well and remained neurologically normal postoperatively. His peritoneal dialysis was managed by the nephrology service. He was discharged home on the 3rd postoperative day and has remained neurologically normal 1 year postoperatively. Repeat cervical CT angiography and MR angiography 1 year after surgical revascularization showed no re-stenosis at the patch site and much better filling of the distal cervical ICA.

\section{Discussion}

Loss-of-function mutations in SMARCAL1 (SWI/SNFrelated, matrix-associated, actin-dependent regulator of chromatin, subfamily a-like 1 ) cause SIOD. ${ }^{4}$ The estimated incidence of SIOD is 1 in $1-3$ million live births. ${ }^{10}$ The SMARCAL1 protein is a chromatin-remodeling enzyme shown to stabilize stalled replication forks and prevent replication-associated DNA damage. ${ }^{9-12}$ The exact mechanism linking lesions at this genetic locus to the clinical syndrome is unknown. It has been hypothesized, however, that replication stress in highly mitotic tissues (e.g., epiphyseal tissue, bone marrow) could play a role. ${ }^{1}$

Cerebrovascular pathology in SIOD could result from several factors. Hypertension and hyperlipidemia secondary to renal disease combined with immune dysfunction could predispose a patient to atherosclerosis, which has been observed in postmortem specimens. ${ }^{6,12}$ Another likely mechanism, recently demonstrated by Morimoto et al., is decreased elastin expression in vascular tissues. ${ }^{8}$ This mechanism is consistent with known microscopic vascular pathology in SIOD, which characteristically shows disorganized internal elastic lamina as well as medial and intimal hyperplasia, and is also consistent with the intraoperative findings in this case report.

In this particular case of an asymptomatic patient with SIOD and high-grade ICA stenosis, 4 management options were considered. Serial observation was ruled out because of the relentless progression of arteriosclerosis and myointimal hyperplasia in SIOD, and the high-risk location of the focal stenosis for causing future strokes. Medical management with antiplatelet or anticoagulant agents was also considered. As a result of the aforementioned progressive nature of the disease, this medical option was believed to be suboptimal. Endovascular stent placement was judged unlikely to provide lasting dilation in the face of what was likely a dense, hyperplastic lesion. Additionally, no guiding experience exists for the use of stents in children. Surgical revascularization was determined to be the best course of action. Unlike adult surgery for atherosclerotic ICA disease, we were doubtful of being able to resect the stenosing myointimal hyperplasia, but believed expansion of the carotid artery lumen could be achieved by placement of a patch graft. Another alternative was excision 
of the stenotic carotid segment and replacement with an inline graft. This was rejected, however, because of the possibility of recurrent stenosis and repeat surgeries. We decided that extending an arteriotomy would be a more simple operation than serial excision of carotid segments with graft replacement. Also, we believed that leaving the native vessel in place would allow for a more normal growth potential.

As recent advances in transplantation and dialysis have increased life expectancy in children with SIOD, cerebrovascular disease has become an increasingly important contributor to morbidity and mortality. In the future, it will be important to consider SIOD as a neurosurgical disease. This case demonstrates that carotid arteriotomy and patching can be safely applied in the setting of SIOD. Furthermore, this case also suggests that this same technique can be used as a treatment option for focal carotid artery narrowing of other origins in pediatric patients.

\section{Acknowledgments}

We thank Elizabeth Hoyte for assistance with figure preparation and Cindy H. Samos for editorial assistance.

\section{References}

1. Bansbach CE, Boerkoel CF, Cortez D: SMARCAL1 and replication stress: an explanation for SIOD? Nucleus 1:245-248, 2010

2. Boerkoel CF, Nowaczyk MJ, Blaser SI, Meschino WS, Weksberg R: Schimke immunoosseous dysplasia complicated by moyamoya phenomenon. Am J Med Genet 78:118-122, 1998

3. Boerkoel CF, O’Neill S, André JL, Benke PJ, Bogdanovíć R, Bulla M, et al: Manifestations and treatment of Schimke immuno-osseous dysplasia: 14 new cases and a review of the literature. Eur J Pediatr 159:1-7, 2000

4. Boerkoel CF, Takashima H, John J, Yan J, Stankiewicz P, Rosenbarker L, et al: Mutant chromatin remodeling protein SMARCAL1 causes Schimke immuno-osseous dysplasia. Nat Genet 30:215-220, 2002

5. Clewing JM, Antalfy BC, Lücke T, Najafian B, Marwedel
KM, Hori A, et al: Schimke immuno-osseous dysplasia: a clinicopathological correlation. J Med Genet 44:122-130, 2007

6. Ehrich JH, Burchert W, Schirg E, Krull F, Offner G, Hoyer $\mathrm{PF}$, et al: Steroid resistant nephrotic syndrome associated with spondyloepiphyseal dysplasia, transient ischemic attacks and lymphopenia. Clin Nephrol 43:89-95, 1995

7. Lücke T, Marwedel KM, Kanzelmeyer NK, Hori A, Offner $\mathrm{G}$, Kreipe HH, et al: Generalized atherosclerosis sparing the transplanted kidney in Schimke disease. Pediatr Nephrol 19:672-675, 2004

8. Morimoto M, Yu Z, Stenzel P, Clewing JM, Najafian B, Mayfield C, et al: Reduced elastogenesis: a clue to the arteriosclerosis and emphysematous changes in Schimke immunoosseous dysplasia? Orphanet J Rare Dis 7:70, 2012

9. Postow L, Woo EM, Chait BT, Funabiki H: Identification of SMARCAL1 as a component of the DNA damage response. J Biol Chem 284:35951-35961, 2009

10. Yusufzai T, Kadonaga JT: HARP is an ATP-driven annealing helicase. Science 322:748-750, 2008

11. Yusufzai T, Kong X, Yokomori K, Kadonaga JT: The annealing helicase HARP is recruited to DNA repair sites via an interaction with RPA. Genes Dev 23:2400-2404, 2009

12. Zieg J, Krepelova A, Baradaran-Heravi A, Levtchenko E, Guillén-Navarro E, Balascakova M, et al: Rituximab resistant evans syndrome and autoimmunity in Schimke immuno-osseous dysplasia. Pediatr Rheumatol Online J 9:27, 2011

\section{Author Contributions}

Conception and design: Steinberg, Westbroek, Kalanithi. Acquisition of data: Westbroek. Analysis and interpretation of data: all authors. Drafting the article: Westbroek, Mukerji. Critically revising the article: all authors. Reviewed submitted version of manuscript: all authors. Approved the final version of the manuscript on behalf of all authors: Steinberg. Study supervision: Steinberg, Mukerji.

\section{Correspondence}

Gary K. Steinberg, Department of Neurosurgery, Stanford University School of Medicine, 300 Pasteur Dr., R-281, Stanford, CA 94305-5327. email: gsteinberg@stanford.edu. 